\title{
A case of dengue fever with acute appendicitis: Not dengue fever mimicking appendicitis
}

\author{
L. Kumarasena, P. Piranavan, S. Bandara, W.P.G. Pubudu, B. Jayasundara, A. de Silva \\ National Hospital of Sri Lanka
}

Keywords: Dengue; appendicitis

\section{Introduction}

Dengue fever (DF) is endemic in Sri Lanka with more than 44,000 reported cases in 2012. It is a common cause for an acute febrile illness and clinical profile ranges from self limiting fever to dengue shock syndrome. Other than for the febrile illness, DF may rarely present as apparent acute abdominal surgical emergency such as acute cholecystitis, acute pancreatitis, and acute appendicitis (AA). Published literature provides limited number of reports of acute abdominal problems mimicking DF. [1-4] Acute appendicitis is a common condition which can get complicated with perforation, peri-appendicial abscess formation, peritonitis and rarely death. [5] We report the experience with an adolescent presented as having AA which was confirmed peri-operatively as well as histologically, who also had evidence of dengue hemorrhagic fever (DHF) during post-operative period. To the best of our knowledge this is the first case of simultaneous AA and DHF, which created a diagnostic and management dilemma.

\section{Case report}

A twelve and a half year old boy was admitted to a medical casualty unit with fever and abdominal pain of one day duration. He was then referred to the surgical team with suspicion of AA. At surgical evaluation the patient had right sided lower abdominal pain and fever for 24 hours. He denied of migrating abdominal pain. He had anorexia, nausea and vomiting but no urinary symptoms. He was febrile $\left(100.5^{\circ} \mathrm{F}\right)$, had a regular pulse rate of 96 beats per minute and was haemodynamically stable. There was no flushing of his body. There was

Correspondence: B. Jayasundara

E-mail: bingumal@sltnet.lk evidence of localized peritonism at right iliac fossa with guarding and rebound tenderness. His urine analysis was normal and white cell count (WCC) was $7.2 \times 106 / 1$ with 79\% neutrophils and 18\% lymphocytes. Platelet count was $195 \times 109 / 1$. With clinical evidence of AA supported by an Alvarado score of $7 / 10$, patient underwent open appendicectomy. Surgical procedure was uncomplicated and the appendix was found to be inflamed. $\mathrm{He}$ was given diclofenac $\mathrm{Na} 25 \mathrm{mg}$ suppository at recovery, followed by regular oral paracetamol and subcutaneous morphine for pain relief. Intravenous cefuroxime and metronidazole was continued for a maximum of three doses. Initial 12 hours of the post-operative period was uncomplicated and the child was commenced on oral feeding. Twenty four hours after appendicectomy, the patient had evidence of increased localized peritonism which gradually progress to be generalized. He developed a tachycardia of 110 beats per minute but the blood pressure was stable. There was no skin rashes noted. Repeated WCC was $3.4 \times 106 / \mathrm{L}$ with $78 \%$ neutrophils and $18 \%$ lymphocytes. Platelet count was $147 \times 109 /$ L. Creactive protein (CRP) level was $23 \mathrm{mg} / \mathrm{L}$ (normal value $<6$ ). Due to worsening peritonism with a suspicion of inadvertent bowel injury or ligature slip the patient had re-exploration through the previous incision 36 hours after appendicectomy. The second exploration was negative and there was no excessive bleeding from the incision. Abdomen was closed after a peritoneal lavage. Subsequently the patient developed further thrombocytopenia and neutropenia around 72 hours from the onset of initial complain, leading to the possible diagnosis of DHF. At this stage the diagnosis was confirmed with a positive Dengue NS1 antigen test. By fifth day of fever he had the maximum thrombocytopenia of $46 \times 109 / \mathrm{L}$ without bleeding manifestations. He had elevated liver transaminase 
levels. The patient was managed at critical care setting with inputs from both medical and surgical teams. He did not require transfusion of blood products. From sixth day, clinical and biochemical parameters did improve and the patient was discharged on day 12 , with normal WCC, platelet count and liver enzyme levels. Histological evaluation confirmed AA with transmural neutrophil infiltration of the appendix.

\section{Discussion}

Although self limiting abdominal pain and manifestations like nausea and/or vomiting, diarrhoea are seen in dengue infection, DF presenting as acute abdomen is rare. Published literature cumulatively report 21 cases of DF presenting as AA, and six patients have undergone appendicectomy. [1-4] Microscopic evaluation of these specimens showed lymphocytic infiltration, lymphoid hyperplasia or normal microscopy. None of these patients had histological evidence of acute appendicitis and this patient is probably the first to have microscopic evidence of supurative AA. Exact mechanism of acute abdomen in DF is not known. Wu et al postulated cholestasis, endotoxemia, microangiopathy, ischaemia and reperfusion injury as possible pathogenic mechanisms for acute acalculous cholecystitis in DF [6]. With the limited number of cases reported, it is still early to apply similar postulations for apparent AA in DF.

Diagnosis of AA and management decision making is mainly on clinical judgment. Clinical diagnosis of appendicitis is also supported by raised inflammatory markers. Alvarado provided a scoring system which counted elevated WCC and left shift in addition to clinical parameters, and most surgeons adapt this system. [7] Despite the general expectation of elevated serum markers like raised WCC and CRP, there are reports of gangrenous appendicitis with normal serum markers. $[8,9]$

This patient highlights the dilemma created by AA with DF on patient management for both surgical and medical teams. Suspicion of DF was delayed until the second exploration as patient had classical features of appendicitis which was apparent at first surgery. Previous cases of acute abdomen with DF which were surgically intervened, required substantial amount of blood/ blood product transfusion. [1, 3] The index patient did not develop such complications. It was unclear whether this patient had simultaneous appendicitis and dengue fever as two different presentations of dengue virus, or as a dual pathology. Previous authors, who have managed the cases of DF mimicking AA, have commented on the need to differentiate the disease early to avoid unnecessary surgical interventions. We highlight the occurrence of true intra-abdominal inflammatory process of appendicitis in the index case, which could have been a rare presentation in DF either as a part of dengue virus infection or as a concurrent dual pathology.

Acknowledgements

We acknowledge the valued contribution of University medical team for their input in the patient management, Dr Janakie Fernando and her team for the histological assessment of the resected specimen and Dr Disna de Silva for her advices in the preparation of the article.

\section{References}

1. Khor BS, Liu JW, Lee IK, Yang KD. Dengue hemorrhagic fever patients with acute abdomen: clinical experience of 14 cases. American Journal of Tropical Medicine and Hygiene 2006; 74: 901-4.

2. Premaratna R, Bailey MS, Ratnasena BGN, de Silva HJ. Dengue fever mimicking acute appendicitis. Transactions of Royal Society of Tropical Medicine and Hygiene 2007; 101: 683-5.

3. Shamim M. Frequency, Pattern and Management of Acute Abdomen in Dengue Fever in Karachi, Pakistan. Asian Journal of Surgery 2010;33: 107-13.

4. Kang YJ, Choi SY, Kang IJ et al. Dengue fever mimicking acute appendicitis: a case report. Infection and Chemotherapy 2009; 41:236-9.

5. Simpson J, Samaraweera AP, Sara RK, Lobo DN. Acute appendicitis - a benign disease? Annals of Royal College of Surgeons of England 2008; 90:313-6.

6. Wu KL, Changchien CS, Kuo CM et al. Dengue fever with acute acalculous cholecystitis. American Journal of Tropical Medicine and Hygiene 2003; 68: 657-60.

7. Alvarado A. A practical score for the early diagnosis of acute appendicitis. Annals of Emergency Medicine. 1986; 15: 557-64. 
8. Vaughan-Shaw P, Richardson C, Lewis M. White cell count and $\mathrm{C}$-reactive protein measurement in patients with possible appendicitis. Annals of Royal College of Surgeons of England 2011;93: 183
9. Vaughan-Shaw PG, Rees JR, Bell E et al. Normal inflammatory markers in appendicitis: evidence from two independent cohort studies. Journal of Royal Society Medicine Short Reports 2011; 2: 43.

\section{Key points:}

- Always consider acute appendicitis, when appropriate, with presentation of other disease

- Alvarado provided a scoring system which counted elevated total leucocyte count and left shift in addition to clinical parameters, and most surgeons adapt this system. 